\title{
Theory of solvation in polar nematics
}

\author{
Vitaly Kapko and Dmitry V. Matyushov \\ Department of Chemistry and Biochemistry and the Center for the Study of Early Events in Photosynthesis, \\ Arizona State University, PO Box 871604, Tempe, AZ 85287-1604
}

(Dated: June 28, 2018)

\begin{abstract}
We develop a linear response theory of solvation of ionic and dipolar solutes in anisotropic, axially symmetric polar solvents. The theory is applied to solvation in polar nematic liquid crystals. The formal theory constructs the solvation response function from projections of the solvent dipolar susceptibility on rotational invariants. These projections are obtained from Monte Carlo simulations of a fluid of dipolar spherocylinders which can exist both in the isotropic and nematic phase. Based on the properties of the solvent susceptibility from simulations and the formal solution, we have obtained a formula for the solvation free energy which incorporates experimentally available properties of nematics and the length of correlation between the dipoles in the liquid crystal. The theory provides a quantitative framework for analyzing the steady-state and time-resolved optical spectra and makes several experimentally testable predictions. The equilibrium free energy of solvation is anisotropic in the nematic phase and is given by a quadratic function of cosine of the angle between the solute dipole and the solvent nematic director. The sign of solvation anisotropy is determined by the sign of dielectric anisotropy of the solvent: solvation anisotropy is negative in solvents with positive dielectric anisotropy and vice versa. The solvation free energy is discontinuous at the point of isotropic-nematic phase transition. The amplitude of this discontinuity is strongly affected by the size of the solute becoming less pronounced for larger solutes. The discontinuity itself and the magnitude of the splitting of the solvation free energy in the nematic phase are mostly affected by microscopic dipolar correlations in the nematic solvent. Illustrative calculations are presented for the Stokes shift and Stokes shift correlation function of coumarin-153 in 4-n-pentyl-4'-cyanobiphenyl (5CB) and 4,4-n-heptyl-cyanopiphenyl $(7 \mathrm{CB})$ solvents as a function of temperature in both the nematic and isotropic phase.
\end{abstract}

\section{INTRODUCTION}

The problem of polar solvation is one of the oldest problems of Physical Chemistry which yet is still a field of active theoretical and experimental research. The calculation of solvation free energy is particularly complex, since it is affected by a variety of contributions including the short-range cavity formation energy, medium-range dispersion and induction forces (non-polar solvation), and long-range electrostatic interactions (polar solvation). These components often compensate and complement each other when solvents of different polarity are considered. As a result, calculations of the overall free energy of solvation are challenging and often require phenomenological parametrization. Many phenomena encountered in chemistry (spectroscopy, redox reactions, etc.) are, however, less affected by the cavitation free energy than by non-polar and polar solvation, while the latter often dominates in polar solvents. Therefore, much effort over the last 80 years, following the work of Born, ${ }^{1}$ Onsager,$\frac{2}{2}$ and Kirkwood ${ }^{3}$ has focused on the understanding and modeling of electrostatic, polar solvation.

The original Born-Onsager idea of calculating the electrostatic solvation free energy as the continuum dielectric response to charges of the solute has found broad applications, in particularly to solvation of large molecules often encountered in bio-medical research $\frac{4.5}{}$ For smaller solutes, formal liquid-state theories, most notably integral equation theories, have found broad application. These theories are normally formulated either in terms of site-site $\mathrm{e}^{\underline{6}}$ or multipolar interaction ${ }^{7}$ potentials. The proliferation of computer simulation techniques has helped to clarify many microscopic features of solvation as well as to test and refine the formal models.

Most of the effort in the field of solvation thermodynamics has been focused on the understanding of solvation in isotropic solvents. Anisotropic solvation, important for chemical reactivity in biological membranes, surfaces, and liquid crystalline solvents, has attracted relatively little attention. Also, from the side of experiment, almost nothing is known about thermodynamics of solvation in liquid crystals. There is a very limited evidence on the solvatochromic shift from spectroscopy $\stackrel{8}{\underline{*}}$ and a few solvation dynamics studies $\stackrel{9.10 .11}{ }$ have been reported. Computer experiment on solvation in liquid crystals virtually does not exist. Continuum models, representing the effect of solvent anisotropy by a tensorial dielectric constant, have been proposed $\stackrel{12.13}{=}$ These approaches provide a very useful continuum limit since,

*E-mail: dmitrym@asu.edu. 
for instance, the Onsager problem of solvation of a spherical dipole ${ }^{2}$ has an exact analytical solution for continuum nematics 14.15

Despite the progress in using dielectric continuum models, a few fundamental problems still need to be resolved. First, limits of the applicability of the continuum approximation to solvation in polar nematics have not been established. Liquid crystals are mostly made of bulky elongated molecules, and it is a priori unclear if continuum models can be applied to solvation of solutes of size often comparable to the size of the solvent molecules. Second, it is not clear if dielectric response of a liquid crystal to the solute electric field can in principle be represented by a single quantity, the dielectric constant, in particular close to the isotropic-nematic phase transition.

The approach we propose in this paper is based on the recently obtained microscopic solution for dipole solvation ${ }^{16}$ The model is based on the assumption that the solute-solvent interaction potential is given by the interaction of the solute charges with the solvent dipolar polarization. The solvation free energy is then expressed through the polarization autocorrelation function of the pure solvent without any particular assumptions regarding the solvent structure. The theory is thus applicable to an arbitrary isotropic dielectric. The goal of this paper is to generalize this approach to the case of a solvent with axial symmetry. For solvation in isotropic liquids, two projections of the polarization autocorrelation function, longitudinal and transverse, are sufficient to describe the dipolar response. Lowering the symmetry of the solvent requires a larger set of projections. We derive a formally exact expression for the free energy of ionic and dipolar solvation in Sec. II [Eq. [46)].

The full formulation of the theory requires projections of the polarization correlation function on rotational invariants. These are obtained here from computer simulations of a fluid of dipolar hard spherocylinders. The application of the theory to experiment requires, however, a solution based on the input parameters available from experiment. This formulation is given in Sec. III] [Eq. [58] in form of a linear combination of solutions obtained in the limit of zero wavenumber (continuum) and infinite wavenumber. The relative contribution of each component depends on the correlation length of dipolar fluctuations in the liquid (distinct from the correlation length of the order parameter fluctuations in Landau-de Gennes theory of liquid crystals17). One of the principle results of this study is a very slow approach of the solvation free energy to its continuum limit, thus invalidating continuum approaches to solvation of small and medium-size solutes. We study the dependence of the free energy of solvation on the angle between the solute dipole and nematic director as well as on temperature when crossing the point of isotropic-nematic transition in Sec. IV The solvation free energy is shown to pass through a discontinuity at the transition temperature and becomes anisotropic in the nematic phase. Also, the Stokes shift correlation function changes from a single-exponential decay in the isotropic phase to bi-exponential decay in the nematic phase. Our results are summarized in Sec. $\mathbf{\nabla}$

\section{THEORY}

The linear response approximation (LRA) provides a solution for the solvation free energy (strictly speaking, the chemical potential) $\mu$ in terms of the response function $\chi\left(\mathbf{r}_{1}, \mathbf{r}_{2}\right)$ which gives the dipolar polarization in the point of space $\mathbf{r}_{1}$ produced by the external electric field $\mathbf{E}_{0}\left(\mathbf{r}_{2}\right)$ at the point of space $\mathbf{r}_{2}$ :

$$
\mathbf{P}\left(\mathbf{r}_{1}\right)=\int \chi\left(\mathbf{r}_{1}, \mathbf{r}_{2}\right) \cdot \mathbf{E}_{0}\left(\mathbf{r}_{2}\right) d \mathbf{r}_{2},
$$

where subscript " 0 " for the variables refer to the solute. The solvation free energy is then

$$
\mu=-\frac{1}{2} \int \mathbf{P}\left(\mathbf{r}_{1}\right) \cdot \mathbf{E}_{0}\left(\mathbf{r}_{1}\right) d \mathbf{r}_{1} .
$$

The dependence on two separate positions, instead of $\mathbf{r}_{1}-\mathbf{r}_{2}$ of homogeneous solvents, reflects the inhomogeneous nature of the solvent response in the presence of the repulsive core of the solute expelling the solvent from its volume.

In k-scape, Eq. (2) becomes

$$
\mu=-\frac{1}{2} \int \frac{d \mathbf{k}_{1} d \mathbf{k}_{2}}{(2 \pi)^{6}} \tilde{\mathbf{E}}_{0}\left(\mathbf{k}_{1}\right) \cdot \tilde{\chi}\left(\mathbf{k}_{1}, \mathbf{k}_{2}\right) \cdot \tilde{\mathbf{E}}_{0}\left(-\mathbf{k}_{2}\right) .
$$

Here, the Fourier transform of the electric field is taken over the solvent volume $\Omega$ excluding the space occupied by the solute

$$
\tilde{\mathbf{E}}_{0}\left(\mathbf{k}_{1}\right)=\int_{\Omega} \mathbf{E}_{0}(\mathbf{r}) e^{i \mathbf{k} \cdot \mathbf{r}} d \mathbf{r} .
$$

The solute space is made by the van der Waals repulsive cores of its atoms. The radii of the solute atoms exposed to the solvent are augmented by the shortest distance to the solvent dipole, which, for cylindrically symmetric molecules, 
is equal to the radius of the cylindrical part of the molecule. Further, the second-rank tensor $\tilde{\chi}$ is

$$
\tilde{\chi}_{\alpha \beta}\left(\mathbf{k}_{1}, \mathbf{k}_{2}\right)=\frac{1}{k_{B} T}\left\langle\delta \tilde{P}_{\alpha}\left(\mathbf{k}_{1}\right) \delta \tilde{P}_{\beta}\left(-\mathbf{k}_{2}\right)\right\rangle_{0},
$$

where $\delta \tilde{\mathbf{P}}(\tilde{\mathbf{k}})$ is the Fourier transform of the fluctuation of the solvent dipolar polarization.

The LRA solution is independent of the electrostatic field of the solute and the subscript "0" in the angular brackets denotes the statistical average taken at the presence of a fictitious solute with the repulsive core of the real solute but the electrostatic solute-solvent coupling turned off ${ }^{16.18}$ In a hypothetical case of an infinitely small solute, $\tilde{\chi}$ is equal to the dipolar susceptibility of pure solvent $\tilde{\chi}_{s}$ which depends on only one wavevector:

$$
\tilde{\chi}\left(\mathbf{k}_{1}, \mathbf{k}_{2}\right)=\delta_{\mathbf{k}_{1}, \mathbf{k}_{2}} \tilde{\chi}_{s}\left(\mathbf{k}_{1}\right),
$$

where subscript "s" denotes the solvent.

In the general case, $\tilde{\chi}\left(\mathbf{k}_{1}, \mathbf{k}_{2}\right)$ is affected by the presence of the solute and depends on two k-vectors. The effect of the solute on solvent response can generally be separated into two major contribution. The repulsive core of the solute distorts the local density of the solvent around it. The spherically-symmetric solute-solvent pair correlation function $h_{0 s}(r)$ is then different from the solvent-solvent pair correlation function $h_{s s}(r)$. This density disturbance affects the dipolar polarization and, consequently, the response function. Another, by far more significant, effect of the solute on the solvent response function is related to the expulsion of the dipolar polarization from the solute volume. In continuum models, this effect is responsible for the surface charge at the dielectric cavity and, when the cavity does not coincide with the equipotential surface, results in a transverse component in the dielectric response. The Maxwell's dielectric displacement ${ }^{19} \mathbf{D}(\mathbf{r})$ then differs from the external electric field $\mathbf{E}_{0}(\mathbf{r})$.

The exclusion of the dipolar polarization from the solute volume is accounted for in Chandler's Gaussian approximation ${ }^{18.20}$ resulting in the following equation for the $\mathbf{k}$-space response function ${ }^{16}$

$$
\tilde{\chi}\left(\mathbf{k}_{1}, \mathbf{k}_{2}\right)=\delta_{\mathbf{k}_{1}, \mathbf{k}_{2}} \tilde{\chi}_{s}\left(\mathbf{k}_{1}\right)-\tilde{\chi}^{\prime \prime}\left(\mathbf{k}_{1}\right) \cdot \tilde{\theta}_{0}\left(\mathbf{k}_{1}-\mathbf{k}_{2}\right) \tilde{\chi}_{s}\left(\mathbf{k}_{2}\right) .
$$

Here, $\delta_{\mathbf{k}_{1}, \mathbf{k}_{2}}=(2 \pi)^{3} \delta\left(\mathbf{k}_{1}-\mathbf{k}_{2}\right)$ and $\tilde{\theta}_{0}(\mathbf{k})$ is the Fourier transform of the step function, which equals to unity inside the solute and is zero everywhere else. Further, in Eq. (5),

$$
\tilde{\chi}^{\prime \prime}(\mathbf{k})=\tilde{\chi}_{s}(\mathbf{k}) \cdot\left[\tilde{\chi}_{s}(\mathbf{k})-\tilde{\chi}^{\prime}(\mathbf{k})\right]^{-1}
$$

where

$$
\tilde{\chi}^{\prime}(\mathbf{k})=\Omega^{-1} \int_{\Omega} d \mathbf{r}_{1} d \mathbf{r}_{2} \chi_{s}\left(\mathbf{r}_{1}-\mathbf{r}_{2}\right) e^{i \mathbf{k} \cdot\left(\mathbf{r}_{2}-\mathbf{r}_{1}\right)}
$$

and integration in Eq. (9) is over the volume $\Omega$ occupied by the solvent.

The substitution of Eq. (17) into Eq. (3) results in the chemical potential of solvation given by the sum of two components:

$$
\mu=\mu_{\mathrm{h}}+\mu_{\mathrm{corr}}
$$

The first term, $\mu_{\mathrm{h}}$, corresponds to the homogeneous response approximation (subscript " $\mathrm{h}$ ") which assumes that correlations of dipolar polarization are not modified by the solute and $\boldsymbol{\chi}\left(\mathbf{k}_{1}, \mathbf{k}_{2}\right)$ can be approximated by dipolar susceptibility of the pure solvent according to Eq. (6). The only modification introduced by the solute is the cutoff of the electric field inside the solute [Eq. (4)]:

$$
-\mu_{\mathrm{h}}=\frac{1}{2} \int \frac{d \mathbf{k}}{(2 \pi)^{3}} \tilde{\mathbf{E}}_{0}(\mathbf{k}) \cdot \tilde{\chi}_{s}(\mathbf{k}) \cdot \tilde{\mathbf{E}}_{0}(-\mathbf{k}) .
$$

In isotropic solvents, the tensor $\tilde{\chi}_{s}$ is diagonal in a coordinate system with one axis taken along $\mathbf{k}$. Its eigenvalues, the longitudinal $\tilde{\chi}_{s}^{L}$ and transverse $\tilde{\chi}_{s}^{T}$ projections, are quite different in the range $\sigma k<2 \pi$ due to the long-range

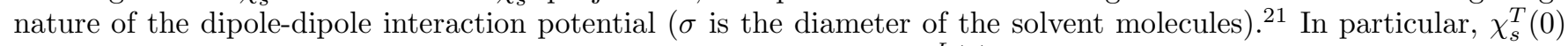
grows as the dielectric constant $\epsilon$ in strongly polar solvents, while $\chi_{s}^{L}(0)$ tends to a constant value. Because of the mutual orthogonality of the longitudinal and transverse projections, the homogeneous solvation free energy $\mu_{\mathrm{h}}$ splits into the longitudinal (L) and transverse $(\mathrm{T})$ parts, each given by a 3D integral in k:

$$
\mu_{\mathrm{h}}=\mu_{\mathrm{h}}^{L}+\mu_{\mathrm{h}}^{T},
$$




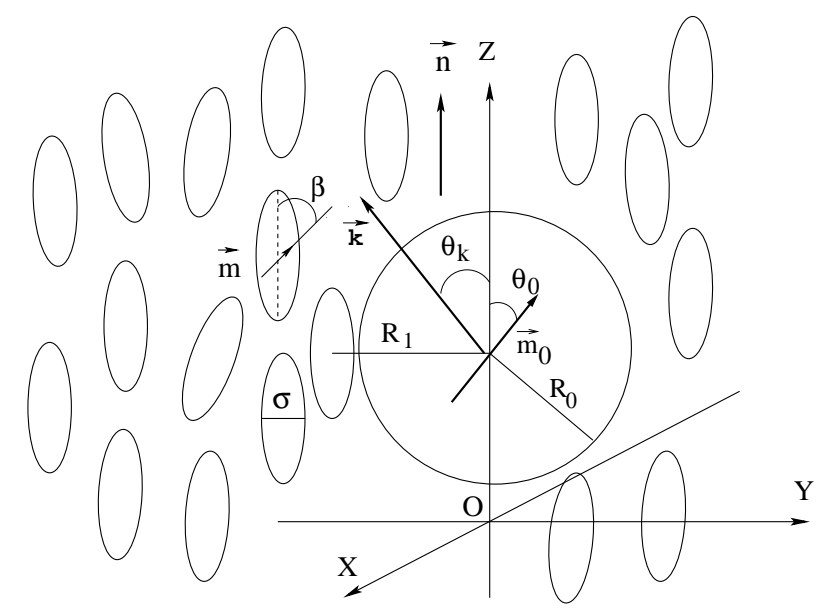

FIG. 1: Dipolar solute in a nematic solvent. The laboratory system of coordinate is chosen to align the $z$-axis wit the nematic director $\hat{\mathbf{n}} . \mathbf{m}_{0}$ denotes the direction of the solute dipole, $\mathbf{k}$ is the wave-vector. $\beta$ is the angle between the dipole moment and the long axis of the solvent molecule.

where

$$
-\mu_{\mathrm{h}}^{L, T}=\frac{1}{2} \int \frac{d \mathbf{k}}{(2 \pi)^{3}} \chi_{s}^{L, T}(k)\left|\tilde{E}_{0}^{L, T}(\mathbf{k})\right|^{2} .
$$

Once the transverse component of the field $\tilde{E}_{0}^{T}(\mathbf{k})$ is non-zero, which happens when either the solute shape or the solute electric field deviates from spherical symmetry, $\mu_{\mathrm{h}}^{T}$ grows linearly with the dielectric constant resulting in the "transverse catastrophe". Therefore, the homogeneous approximation gives reasonable results only for spherical ions when the transverse component of dipolar response is eliminated by the symmetry. It is the second term, generally represented by a $6 \mathrm{D}$ integral over the two k-vectors, that corrects (subscript "corr") for the unphysical behavior of the transverse response.

The correction component $\mu_{\text {corr }}$ can be evaluated exactly using analytical properties of the response function when the electric field is known in the analytical form. In case of a dipole solute, the final solution is conveniently formulated in terms of $\mu_{\mathrm{h}}^{L, T}$ (Ref. 16):

$$
\mu=\left(\chi_{\mathrm{tr}}\right)^{-1}\left[\chi_{s}^{T}(0) \mu_{\mathrm{h}}^{L}+\chi_{s}^{L}(0) \mu_{\mathrm{h}}^{T}\right]
$$

where

$$
\chi_{\mathrm{tr}}=\frac{1}{3}\left(\chi_{s}^{L}(0)+2 \chi_{s}^{T}(0)\right) .
$$

Unfortunately, this scheme is hard to implement for liquid crystals. Since a liquid crystal has its own symmetry axis (Fig. (1), $\tilde{\chi}_{s}$ needs to be diagonalized for each value of $\mathbf{k}$ making the problem rather complex. Expansion of the solvent response function in spherical harmonics ${ }^{22,23}$ appears to be a more straightforward way to the solution. Our solution below is given for the case of spherical ion ("i") and spherical dipole ("d"). These two solutes are characterized by the following expressions for the field Fourier transform in Eq. (4)

$$
\tilde{\mathbf{E}}_{0}^{(i)}(\mathbf{k})=4 \pi i q_{0} \frac{j_{0}\left(k R_{1}\right)}{k} \hat{\mathbf{k}}
$$

and

$$
\tilde{\mathbf{E}}_{0}^{(d)}(\mathbf{k})=-4 \pi \frac{j_{1}\left(k R_{1}\right)}{k R_{1}} \mathbf{m}_{0} \cdot \hat{\mathbf{D}}_{\mathbf{k}}
$$

In Eqs. (16) and (17), $q_{0}$ and $\mathbf{m}_{0}$ are the solute charge and dipolar moment, and $\hat{\mathbf{D}}_{\mathbf{k}}=3 \hat{\mathbf{k}} \hat{\mathbf{k}}-\mathbf{1}$ is the dipolar tensor. Further, $R_{0}$ is the solute radius, $R_{1}=R_{0}+\sigma / 2$ is the distance of closest solute-solvent separation, and $\hat{\mathbf{k}}=\mathbf{k} /|\mathbf{k}|$. Here we also use the standard notation for the spherical Bessel functions $j_{l}(x)$. 
The correction term in Eq. (10) is given by a double k-integral

$$
\mu_{\text {corr }}=\frac{1}{2} \int \frac{d \mathbf{k}_{1} d \mathbf{k}_{2}}{(2 \pi)^{6}} \tilde{\mathbf{E}}_{0}\left(\mathbf{k}_{1}\right) \cdot \tilde{\chi}^{\prime \prime}\left(\mathbf{k}_{1}\right) \cdot \tilde{\theta}_{0}\left(\mathbf{k}_{1}-\mathbf{k}_{2}\right) \tilde{\chi}_{s}\left(\mathbf{k}_{2}\right) \cdot \tilde{\mathbf{E}}_{0}\left(-\mathbf{k}_{2}\right)
$$

In order co convert it to a computationally tractable 3D integral, we first introduce a direct-space field

$$
\mathbf{F}_{0}(\mathbf{r})=\int \frac{d \mathbf{k}}{(2 \pi)^{3}} e^{-i \mathbf{k} \cdot \mathbf{r}} \tilde{\mathbf{E}}_{0}(\mathbf{k}) \cdot \tilde{\chi}^{\prime \prime}(\mathbf{k}) .
$$

Analytic properties of the response function $\tilde{\chi}^{\prime \prime}$ in complex $k$-plane allows one to reduce the integration over $\mathbf{k}$ to the angular integral over the directions of $\mathbf{k}^{16}$ For the field of a spherical dipole, $\mathbf{F}_{0}=\mathbf{F}^{(d)}$ is constant within the solute

$$
\mathbf{F}^{(d)}=-\frac{1}{R_{1}^{3}} \int \frac{d \omega_{k}}{4 \pi} \mathbf{m}_{0} \cdot \hat{\mathbf{D}}_{\mathbf{k}} \cdot \tilde{\chi}^{\prime \prime}(k=0)
$$

For the spherical ion (see below),

$$
\mathbf{F}^{(i)}=\mathbf{F}_{0}=0
$$

In order to calculate $\mathbf{F}_{0}$ in Eq. (20), we need to obtain $\tilde{\chi}^{\prime \prime}(k=0)$ from the $k=0$ value of the solvent dipolar susceptibility, which we consider next.

\section{A. Continuum limit}

We use the laboratory Cartesian system of coordinates with $z$-axis parallel to the nematic director $\hat{\mathbf{n}}$ (Fig. (1). The continuum limit for solvent response function can be obtained from Maxwell's material equations with axially symmetric dielectric constant characterized by longitudinal $\left(\epsilon_{\|}=\epsilon_{z}\right)$ and transverse $\left(\epsilon_{\perp}=\epsilon_{x}=\epsilon_{y}\right)$ components: ${ }^{24}$

$$
4 \pi \tilde{\chi}_{s, \alpha \beta}(k=0)=\left(\epsilon_{\alpha}-1\right) \delta_{\alpha \beta}-\frac{\hat{k}_{\alpha} \hat{k}_{\beta}\left(\epsilon_{\alpha}-1\right)\left(\epsilon_{\beta}-1\right)}{\epsilon_{\perp}+\left(\epsilon_{\|}-\epsilon_{\perp}\right)(\hat{\mathbf{k}} \cdot \hat{\mathbf{n}})^{2}} .
$$

Note that $\tilde{\chi}_{s}(k=0)$ is an even function of $\hat{\mathbf{k}}$; therefore, according to Eqs. (16) and (19), $\mathbf{F}_{0}=0$ for the spherical ion [Eq. [21]].

To proceed with the dipolar solute, we first calculate the integral

$$
A_{\alpha \beta}=-\int \frac{d \omega_{k}}{4 \pi} \sum_{\gamma} \hat{D}_{\mathbf{k}, \alpha \gamma} \tilde{\chi}_{s, \gamma \beta}(k=0) .
$$

The matrix $\mathbf{A}$ is diagonal with the elements:

$$
\begin{gathered}
A_{x x}=A_{y y}=\frac{\epsilon_{\perp}-1}{8 \pi\left(\epsilon_{\|}-\epsilon_{\perp}\right)}\left[\left(2 \epsilon_{\|}-\epsilon_{\perp}+2\right)-\frac{\epsilon_{\|}}{\epsilon_{\perp}}\left(\epsilon_{\perp}+2\right) \psi\right], \\
A_{z z}=-\frac{\epsilon_{\|}-1}{4 \pi\left(\epsilon_{\|}-\epsilon_{\perp}\right)}\left[\epsilon_{\perp}+2-\left(\epsilon_{\|}+2\right) \psi\right],
\end{gathered}
$$

where

$$
\psi=\int_{0}^{1} \frac{d z}{1+\left(\epsilon_{\|} / \epsilon_{\perp}-1\right) z^{2}}=\left\{\begin{array}{cl}
\arctan \sqrt{\epsilon_{\|} / \epsilon_{\perp}-1} / \sqrt{\epsilon_{\|} / \epsilon_{\perp}-1} & , \epsilon_{\|}>\epsilon_{\perp} \\
1 & , \epsilon_{\|}=\epsilon_{\perp} \\
\ln \left(\frac{1+\sqrt{1-\epsilon_{\|} / \epsilon_{\perp}}}{1-\sqrt{1-\epsilon_{\|} / \epsilon_{\perp}}}\right) /\left(2 \sqrt{1-\epsilon_{\|} / \epsilon_{\perp}}\right) & , \epsilon_{\|}<\epsilon_{\perp} .
\end{array}\right.
$$

Note that $A_{x x}$ and $A_{z z}$, as well as $\tilde{\chi}_{0, x x}$ and $\tilde{\chi}_{0, z z}$ below, have no singularities at $\epsilon_{\|}=\epsilon_{\perp}$ because terms in the square brackets are proportional to $\epsilon_{\|}-\epsilon_{\perp}$ at $\epsilon_{\|}-\epsilon_{\perp} \ll 1$. 
In the Appendix, we prove the relation

$$
\tilde{\chi}_{0} \equiv \tilde{\chi}_{s}(k=0)-\tilde{\chi}^{\prime}(k=0)=\int \frac{d \omega_{k}}{4 \pi} \tilde{\chi}_{s}(k=0) .
$$

Then $\tilde{\chi}_{0}$ is diagonal with the elements

$$
\tilde{\chi}_{0, x x}=\tilde{\chi}_{0, y y}=\frac{\epsilon_{\|}-1}{8 \pi\left(\epsilon_{\|}-\epsilon_{\perp}\right)}\left[\left(2 \epsilon_{\|}-\epsilon_{\perp}-1\right)-\frac{\epsilon_{\|}}{\epsilon_{\perp}}\left(\epsilon_{\perp}-1\right) \psi\right]
$$

and

$$
\tilde{\chi}_{0, z z}=-\frac{\epsilon_{\|}-1}{4 \pi\left(\epsilon_{\|}-\epsilon_{\perp}\right)}\left[\epsilon_{\perp}-1-\left(\epsilon_{\|}-1\right) \psi\right] .
$$

When Eqs. (22) and (27) are used in the definition of $\chi^{\prime \prime}$ in Eq. (8), the final result for the field $\mathbf{F}^{(d)}$ becomes

$$
F_{\alpha}^{(d)}=\frac{m_{0, \alpha}}{R_{1}^{3}} R_{\alpha}
$$

where $\alpha$ stands for $x, y, z$ and

$$
R_{\alpha}=\frac{\epsilon_{\alpha}-n_{\alpha}\left(\epsilon_{\alpha}+2\right)}{\epsilon_{\alpha}-n_{\alpha}\left(\epsilon_{\alpha}-1\right)}
$$

In Eq. (31), the so-called depolarization factors are given by ${ }^{25}$

$$
\begin{aligned}
& n_{z}=\frac{\epsilon_{\|}(\psi-1)}{\epsilon_{\perp}-\epsilon_{\|}}, \\
& n_{x}=n_{y}=\frac{1-n_{z}}{2} .
\end{aligned}
$$

In the isotropic limit, when $\epsilon_{\|}=\epsilon_{\perp}$, one gets $n_{x}=n_{y}=n_{z}=1 / 3$.

The field $\mathbf{F}_{0}$ defined by Eq. (19) is a generalization of the reaction field, introduced by Onsager for a point dipole,, 2 to an arbitrary configuration of solute charges in a solute of arbitrary shape. We have shown here that this field reduces to expected limits in the case of spherical ionic and dipolar solutes. In the former case, the reaction potential created by the polar liquid within the cavity is constant, and the reaction field is zero. In the latter case, the field is constant and our expression in Eqs. (30)-(32) coincides with the reaction field in an axially anisotropic dielectric previously derived for a spherical dipole by solving the Poisson equation $\underline{\underline{15}}$

The zero reaction field in the case of a spherical ion eliminates the correction term in Eq. (10). This means that the solvent response is longitudinal and the dielectric displacement $\mathbf{D}$ is equal to the external field $\mathbf{E}_{0}$. The free energy of solvation is then fully determined by the homogeneous solvation term [Eq. (11)]. In case of a dipole, the solvent response includes a transverse component, the dielectric displacement is not equal to the external field, and the correction term is necessary:

$$
\mu_{\mathrm{corr}}=\frac{1}{2 R_{1}^{3}} \sum_{\alpha \beta} R_{\alpha} m_{0, \alpha} \int \frac{d \mathbf{k}}{(2 \pi)^{3}} \tilde{\chi}_{s, \alpha \beta}(\mathbf{k}) \tilde{\theta}_{0}(\mathbf{k}) \tilde{E}_{0, \beta}(-\mathbf{k}),
$$

where for a spherical solute

$$
\tilde{\theta}_{0}(k)=4 \pi R_{1}^{3} \frac{j_{1}\left(k R_{1}\right)}{k R_{1}} .
$$

Equations (11) and (33) give the correct continuum limit (subscript "c") for the solvation free energy after the replacement of $\tilde{\chi}_{s}(k)$ with its value at $k=0$ :

$$
\mu_{\mathrm{c}}^{(i)}=-\frac{q^{2}}{2 R_{1}}\left(1-\frac{\psi}{\epsilon_{\perp}}\right)
$$

for the ion and

$$
\mu_{\mathrm{c}}^{(d)}=-\frac{1}{2} \mathbf{m}_{0} \cdot \mathbf{F}_{0}^{(d)}=-\frac{m_{0}^{2}}{2 R_{1}^{3}}\left[R_{x}+\left(R_{z}-R_{x}\right) \cos ^{2} \theta_{0}\right]
$$


for the dipole. In Eq. (36), $\theta_{0}$ is the angle between the solute dipolar moment and the director (Fig. (1). In the limit $\epsilon_{\|} \rightarrow \epsilon_{\perp}$, Eqs. (35) and (36) reduce to their well-know isotropic counterparts, the Born formula 1

$$
\mu_{\mathrm{B}}^{(i)}=-\frac{q^{2}}{2 R_{1}}\left(1-\frac{1}{\epsilon}\right)
$$

and the Onsager formula 2

$$
\mu_{\mathrm{O}}^{(d)}=-\frac{m_{0}^{2}}{R_{1}^{3}} \frac{\epsilon-1}{2 \epsilon+1} .
$$

Note that the cavity radius is not specified in continuum models. However, empirical experience suggests using the van der Waals radius $R_{0}$ in place of the radius of closest solute-solvent approach $R_{1}$ appearing in microscopic solvation models.

\section{B. Microscopic theory}

The dependence on the orientation of the wave-vector in Eqs. (11) and (33) can be integrated out by expanding the solvent dipolar susceptibility $\tilde{\chi}_{s}$ in spherical harmonics [Eq. (A44)]:

$$
\tilde{\chi}_{s, n_{1} n_{2}}(\mathbf{k})=\sum_{l} \tilde{\chi}_{s, n_{1} n_{2} l}(k) Y_{l,-n_{1}-n_{2}}^{*}\left(\omega_{k}\right),
$$

where $\omega_{k}$ denotes the orientation of $\hat{\mathbf{k}}$. The spherical components of the solute electric field can be obtained for the ionic and dipolar solutes: ${ }^{22}$

$$
\tilde{E}_{0, n_{1}}^{(i)}(\mathbf{k})=4 \pi \sqrt{\frac{4 \pi}{3}} q_{0} i \frac{j_{0}\left(k R_{1}\right)}{k} Y_{1, n_{1}}^{*}\left(\omega_{k}\right)
$$

and

$$
\tilde{E}_{0, n_{1}}^{(d)}(\mathbf{k})=-16 \pi^{2} \sqrt{\frac{2}{5}} m_{0} \frac{j_{1}\left(k R_{1}\right)}{k R_{1}} \sum_{n_{1}^{\prime}} C\left(112 ; n_{1}, n_{1}^{\prime}, n_{1}+n_{1}^{\prime}\right) Y_{1, n_{1}^{\prime}}\left(\hat{m}_{0}\right) Y_{2, n_{1}+n_{1}^{\prime}}^{*}\left(\omega_{k}\right) .
$$

In Eq. (41), $C\left(l_{1} l_{2} l ; n_{1}, n_{2}, n\right)$ are the Clebsch-Gordan coefficients ${ }_{22}^{22}$ Using the product rule and orthogonality of spherical harmonics ${ }^{22}$ we obtain

$$
\begin{aligned}
\mu_{h}^{(i)} & =-4 q_{0}^{2} \sum_{n_{1} n_{2} l} \int_{0}^{\infty} d k j_{0}^{2}\left(k R_{1}\right) \chi_{s, n_{1} n_{2} l}(k)(-1)^{n_{1}+n_{2}} \frac{C(11 l ; 0,0,0)}{2 l+1} C\left(11 l ; n_{1}, n_{2}, n_{1}+n_{2}\right), \\
\mu_{h}^{(d)} & =-\frac{24 m_{0}^{2}}{R_{1}^{2}} \int_{0}^{\infty} d k j_{1}^{2}\left(k R_{1}\right) \sum_{n 1 n_{2} l} \chi_{s, n_{1} n_{2} l}(k)(-1)^{n_{1}+n_{2}} \frac{C(22 l ; 000)}{2 l+1} \\
& \sum_{n^{\prime}} C\left(112 ; n_{1}, n^{\prime}, n_{1}+n^{\prime}\right) C\left(112 ; n_{2},-n^{\prime}, n_{2}-n^{\prime}\right) C\left(22 l ; n_{1}+n^{\prime}, n_{2}-n^{\prime}, n_{1}+n_{2}\right) \hat{m}_{0, n^{\prime}} \hat{m}_{0,-n^{\prime}}, \\
\mu_{\mathrm{corr}}^{(d)} & =-\frac{4 \sqrt{6} m_{0}^{2}}{5 R_{1}^{2}} \sum_{n_{1} n_{2}} \int_{0}^{\infty} d k j_{1}^{2}\left(k R_{1}\right) \tilde{\chi}_{s, n_{1} n_{2} 2}(k)(-1)^{n_{2}} C\left(112 ; n_{1}, n_{2}, n_{1}+n_{2}\right) \hat{m}_{0, n_{1}}\left(\hat{m}_{0} R\right)_{-n_{1}}, \\
\mu_{\mathrm{corr}}^{(i)} & =0 .
\end{aligned}
$$

In Eq. (42),

$$
\chi_{s, n_{1} n_{2} l}(k)=\sqrt{\frac{2 l+1}{4 \pi}} \tilde{\chi}_{s, n_{1} n_{2} l}(k),
$$


and the relations between the spherical and Cartesian components of the vector $\hat{\mathbf{m}}=\mathbf{m} / m$ are

$$
\begin{aligned}
\hat{m}_{0,0} & =\hat{m}_{0, z}, \\
\hat{m}_{0,1} & =-\left(\hat{m}_{0, x}+i \hat{m}_{0, y}\right) / \sqrt{2}, \\
\hat{m}_{0,-1} & =\left(\hat{m}_{0, x}-i \hat{m}_{0, y}\right) / \sqrt{2} .
\end{aligned}
$$

Similarly, for the second-rank tensor, one has

$$
\begin{aligned}
\left(\hat{m}_{0} R\right)_{0} & =\hat{m}_{0, z} R_{z}, \\
\left(\hat{m}_{0} R\right)_{1} & =-\left(\hat{m}_{0, x}+i \hat{m}_{0, y}\right) R_{x} / \sqrt{2}, \\
\left(\hat{m}_{0} R\right)_{-1} & =\left(\hat{m}_{0, x}-i \hat{m}_{0, y}\right) R_{x} / \sqrt{2} .
\end{aligned}
$$

Similar relations exist for the components of the second-rank tensor $\tilde{\chi}_{s}(\mathbf{k}) 2_{2}^{22}$

For weakly polar solvents the Cartesian components of $\tilde{\chi}_{s}(\mathbf{k})$ form a diagonal matrix in the laboratory system of coordinates specified in Fig. 1 Moreover, the components related to axes $x$ and $y$ are almost equal to each others. In this approximation the solvation free energy reduces to

$$
\begin{aligned}
& \mu_{\mathrm{h}}^{(i)}=-\frac{4 q_{0}^{2}}{3} \int_{0}^{\infty} d k j_{0}^{2}\left(k R_{1}\right) A(k), \\
& \mu_{\mathrm{h}}^{(d)}=-\frac{4 m_{0}^{2}}{5 R_{1}^{2}} \int_{0}^{\infty} d k j_{1}^{2}\left(k R_{1}\right)\left[B(k)+C(k) \cos ^{2} \theta_{0}\right], \\
& \mu_{\text {corr }}^{(d)}=\frac{4 m_{0}^{2}}{5 R_{1}^{2}} \int_{0}^{\infty} d k j_{1}^{2}\left(k R_{1}\right)\left[R_{x} \chi_{s, x x 2}(k)-\left(2 R_{z} \chi_{s, z z 2}(k)+R_{x} \chi_{s, x x 2}(k)\right) \cos ^{2} \theta_{0}\right], \\
& \mu_{\text {corr }}^{(i)}=0,
\end{aligned}
$$

where

$$
\begin{aligned}
& A(k)=2 \chi_{s, x x 0}(k)+\chi_{s, z z 0}(k)+\frac{2}{5}\left(\chi_{s, z z 2}(k)-\chi_{s, x x 2}(k)\right), \\
& B(k)=3 \chi_{s, z z 0}(k)+7 \chi_{s, x x 0}(k)+\frac{1}{7}\left(3 \chi_{s, z z 2}(k)-10 \chi_{s, x x 2}(k)\right)-\frac{4}{7}\left(\chi_{s, z z 4}(k)-\chi_{s, x x 4}(k)\right), \\
& C(k)=\chi_{s, z z 0}(k)-\chi_{s, x x 0}(k)+\frac{1}{7}\left(5 \chi_{s, z z 2}(k)+16 \chi_{s, x x 2}(k)\right)+\frac{12}{7}\left(\chi_{s, z z 4}(k)-\chi_{s, x x 4}(k)\right) .
\end{aligned}
$$

In Eqs. (46) and (47), $\chi_{s, \alpha \alpha l}(k)$ are the coefficients of expansion of the solvent response function in Legendre polynomials $P_{l}\left(\cos \theta_{k}\right)$ :

$$
\tilde{\chi}_{s, \alpha \alpha}(\mathbf{k})=\sum_{l} \chi_{s, \alpha \alpha l}(k) P_{l}\left(\cos \theta_{k}\right)
$$

\section{RESULTS}

The theory developed in the previous section requires static dielectric constants and dipolar susceptibility of the nematic solvent as input. Here we obtain these data from Monte Carlo (MC) simulation of hard spherocylinders with embedded point dipoles ${ }^{26}$ This fluid transforms from isotropic to nematic phase with decreasing density ${ }^{27}$ NVT MC simulations of $N=800$ hard spherocylinders were carried out in our previous study ${ }^{26}$ The dipole moment $\mathbf{m}$ is parallel to the cylinder axis and the aspect ratio of the length $L$ of the cylindrical part of the molecule to its diameter $\sigma$ is equal to 5 . The thermodynamic state of this fluid is fully defined by two parameters: the reduced dipole moment $\left(m^{*}\right)^{2}=m^{2} /\left(k_{B} T \sigma^{3}\right)$ and the packing fraction $\eta=(\pi / 6) \rho \sigma^{3}(1+3 L / 2 \sigma)$, where $\rho=N / V$ is the solvent number density. Details of the simulation protocol are given in Ref. 26 .

Simulations of nematics with high magnitudes of the dipole moment $m^{*}$ are hindered by the tendency of neighboring dipoles to orient in a locally antiferroelectric order. Combined with the elongated shape of the spherocylinders, local antiparallel alignment of dipoles creates bottlenecks in the system phase space, which are hard to explore by 
standard simulation techniques ${ }^{27}$ In addition, the fluid of dipolar spherocylinders becomes smectic at $\left(m^{*}\right)^{2}>6.0$ and $\eta=0.47$. Because of the relatively high aspect ratio of the solvent molecules, the dipole moment in the range $0 \leq\left(m^{*}\right)^{2} \leq 6.0$ gives a relatively small overall density of dipoles and, therefore, a low dielectric constant. As a result, the capabilities of simple hard-core models are rather limited in exploring high-polarity nematics. The structure of real polar nematic liquids, which can demonstrate rather high dielectric constants, $\stackrel{28}{2}$ is mostly determined by dispersion site-site interactions between elongated molecules.

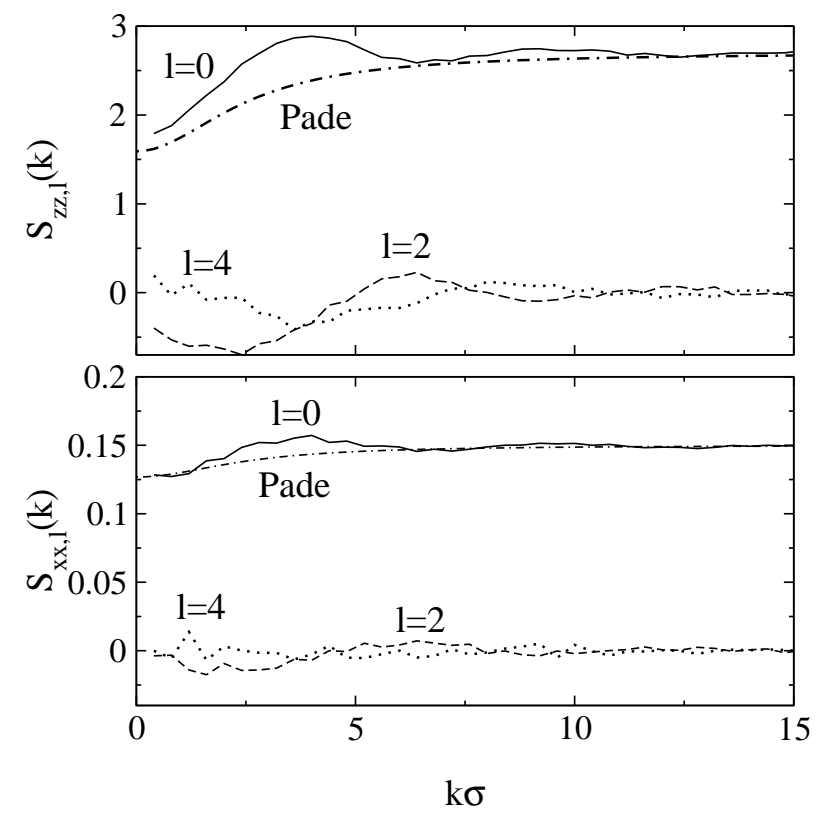

FIG. 2: $S_{z z, l}(k)$ (upper panel) and $S_{x x, l}(k)$ (lower panel) projections of dipolar structure factors for the fluid of hard spherocylinders: $l=0$ (solid line), $l=2$ (dashed line) and $l=4$ (dotted line). The dash-dotted lines refer to the Padé approximation [Eq. (58)] with $\Lambda=0.4 \sigma$. The structure factors are obtained from NVT MC simulations ${ }^{26}$ of 800 solvent molecules with the packing density $\eta=0.47$ and the dipole moment $m^{2} /\left(k_{\mathrm{B}} T \sigma^{3}\right)=1.0$. The fluid is in the nematic phase with the nematic order parameter $S_{2}=0.85$.

For weakly polar nematics, the tensor of the dipolar susceptibility $\tilde{\chi}_{s}$ is nearly diagonal in the Cartesian coordinate system specified in Fig. 11 with almost equal $x x$ and $y y$ projections. Therefore, the formally exact formulas in Eq. (42) can be replaced by the approximate relation in Eq. (46). The solvation free energy then depends on six one-dimensional projection $\chi_{s, \alpha \alpha l}(k)[\mathrm{Eq}$. [48)]. The corresponding structure factors of dipolar polarization

$$
S_{\alpha \alpha, l}(k)=(4 \pi / 3 y) \chi_{s, \alpha \beta l}(k)
$$

have been obtained here from equilibrium MC configurations of the fluid of dipolar spherocylinders (Fig. 2) ${ }^{26}$ In Eq. (49),

$$
y=4 \pi m^{2} \rho /\left(9 k_{B} T\right)
$$

is the standard dipole density parameter of dielectric theories ${ }^{19}$ The reduced dipole moment and the packing fraction of the system have values $\left(m^{*}\right)^{2}=1$ and $\eta=0.47$ (the isotropic-nematic phase transition occurs at $\eta_{\mathrm{IN}} \approx 0.407$ ). In this thermodynamic state, the nematic order parameter $S_{2}$ is equals to 0.85 , and the longitudinal ( $\epsilon_{\|}$, parallel to the director) and transverse ( $\epsilon_{\perp}$, perpendicular to the director) dielectric constants are 1.89 and 1.06 , respectively.

The noise in the structure factors obtained from simulations (Fig. 2) arises from the fluctuations of the nematic director in the laboratory system of coordinates attached to the simulation box. This setup is necessary to insure that the wavevectors used to calculate $S_{\alpha \alpha, l}(k)$ are eigenvectors of the periodic replicas of the cubic simulation cell. The calculations show that the longitudinal structure factors $\left(S_{z z, l}\right)$ are significantly larger than the transverse structure factors $\left(S_{x x, l}\right)$, as expected for nematics with longitudinal dipolar moment. In addition, the magnitudes of projections decrease rapidly with increasing index $l$.

Exact analytical expressions are available for the polarization structure factors in $k \rightarrow 0$ and $k \rightarrow \infty$ limits. The continuum limit relates $S_{z z, l}(0)$ and $S_{x x, l}(0)$ to the anisotropic static dielectric constants through Eqs. (22), (48), and (49). To calculate the $k \rightarrow \infty$ limit, we note that the Cartesian components of the dipolar structure factors are given 
by the following expression

$$
S_{\alpha \beta}(\mathbf{k})=(3 / N) \sum_{i j}\left\langle\hat{m}_{i, \alpha} \hat{m}_{j, \beta} e^{-i \mathbf{k} \cdot \mathbf{r}_{i j}}\right\rangle,
$$

where $N$ is a number of solvent particles. All terms in Eq. (51) with $r_{i j} \neq 0$ vanish at $k \rightarrow \infty$ resulting in

$$
S_{\alpha \beta}(k \rightarrow \infty)=(3 / N) \sum_{i}\left\langle\hat{m}_{i, \alpha} \hat{m}_{i, \beta}\right\rangle=3\left\langle\hat{m}_{\alpha} \hat{m}_{\beta}\right\rangle .
$$

This yields

$$
\begin{aligned}
& S_{x x}(k \rightarrow \infty)=S_{y y}(k \rightarrow \infty)=1-S_{2} P_{2}(\cos \beta), \\
& S_{z z}(k \rightarrow \infty)=1+2 S_{2} P_{2}(\cos \beta), \\
& S_{z x}(k \rightarrow \infty)=S_{z y}(k \rightarrow \infty)=S_{x y}(k \rightarrow \infty)=0,
\end{aligned}
$$

where $\beta$ is the angle between the dipole moment and the long molecular axis of the solvent molecule (Fig. 1) and $P_{2}(x)$ is the second Legendre polynomial.

From Eqs. (46), (47), (49), (52), and (53), we find the expression for the solvation free energy when the $k \rightarrow \infty$ limit is used for the solvent susceptibility:

$$
\mu_{\infty}^{(i)}=-3 y \frac{q_{0}^{2}}{2 R_{1}}
$$

and

$$
\mu_{\infty}^{(d)}=-\frac{m_{0}^{2} y}{R_{1}^{3}}\left(1+\frac{1}{5} S_{2} P_{2}(\cos \beta) P_{2}\left(\cos \theta_{0}\right)\right) .
$$

The continuum $[k=0$, Eqs. (35) and [36] $]$ and short wave-length $[k \rightarrow \infty$, Eqs. (54) and (55)] limits are two asymptotes for the solvation free energy obtained by setting, respectively, the constant $\boldsymbol{\chi}_{s}(0)$ and $\boldsymbol{\chi}_{s}(\infty)$ values for the solvent susceptibility in the $k$-integrals in Eqs. (11) and (33). The advantage of these limiting expressions is their simplicity and direct connection to experimentally available properties of liquid crystalline solvents. These limits can be used to derive a practically useful analytical formula for $\mu$. The largest contribution to $\mu$ comes from the region of $k$ where the squared spherical Bessel functions $j_{l}\left(k R_{1}\right)$ in Eq. (46) has a maximum. This is the region around $k=0$ for the ionic solute and $k \approx 2 / R_{1}$ for the dipolar solute. We need, therefore, a continuous approximation for the structure factors that generates a weighted linear combination of $k=0$ and $k \rightarrow \infty$ limits for the solvation free energy.

The projections of the solvent susceptibility on spherical harmonic are smooth functions of the wavevector for weakly polar nematics. The Cartesian components of the dipolar structure factors can, therefore, be reasonably well approximated by Padé forms interpolating between the $k=0$ and $k \rightarrow \infty$ limits

$$
S_{\alpha \beta, l}(k)=\frac{S_{\alpha \beta, l}(0)+S_{\alpha \beta, l}(\infty) \Lambda_{l}^{2} k^{2}}{1+\Lambda_{l}^{2} k^{2}} .
$$

This formula introduces a new theory parameter, the polarization correlation length $\Lambda_{l}$. The correlation length can be extracted from structure factors obtained from computer simulations by fitting the slope of $S_{\alpha \beta, l}(k)$ vs $k^{2}$ to the $k \rightarrow 0$ expansion of Eq. (56) $\left(k<k_{\max } \simeq 2 / \sigma\right)$. Values of $\Lambda_{l}$ for the fluid of dipolar hard spherocylinders depending on packing fraction $\eta$ and dipolar density $y$ are shown in Fig. 3 (closed points). $\Lambda_{l}$ was found to be rather weakly dependent on $\eta$, even through the isotropic-nematic phase transition (Fig. 3] upper panel). The dependence on $y$ was obtained at fixed packing fraction $\eta=0.432$ and $\left(m^{*}\right)^{2}$ changing from 1.0 to 6.0.

We compare these results to the correlation lengths extracted from slopes of the longitudinal structure factor $S^{L}(k)$ vs $k^{2}$ and the inverse structure factor $1 / S^{T}(k)$ vs $k^{2}$. The latter definition corresponds to the Ornstein-Zernike-Debye plot used for the scattering function of liquids close to the critical temperature when the structure factor is a decaying function of $k \stackrel{29}{2}$ On the contrary, the longitudinal structure factor is a rising function of $k$ requiring the direct expansion of $S^{L}(k)$ in $k^{2}$. The correlation lengths for longitudinal and transverse dipolar fluctuations in isotropic liquids are substantially different. This is because these two projections mix together harmonics of the pair distribution function with different index $l$

$$
\begin{aligned}
& S^{L}(k)=1+\frac{\rho}{3}\left(\tilde{h}^{110}(k)+2 \tilde{h}^{112}(k)\right), \\
& S^{T}(k)=1+\frac{\rho}{3}\left(\tilde{h}^{110}(k)-\tilde{h}^{112}(k)\right) .
\end{aligned}
$$



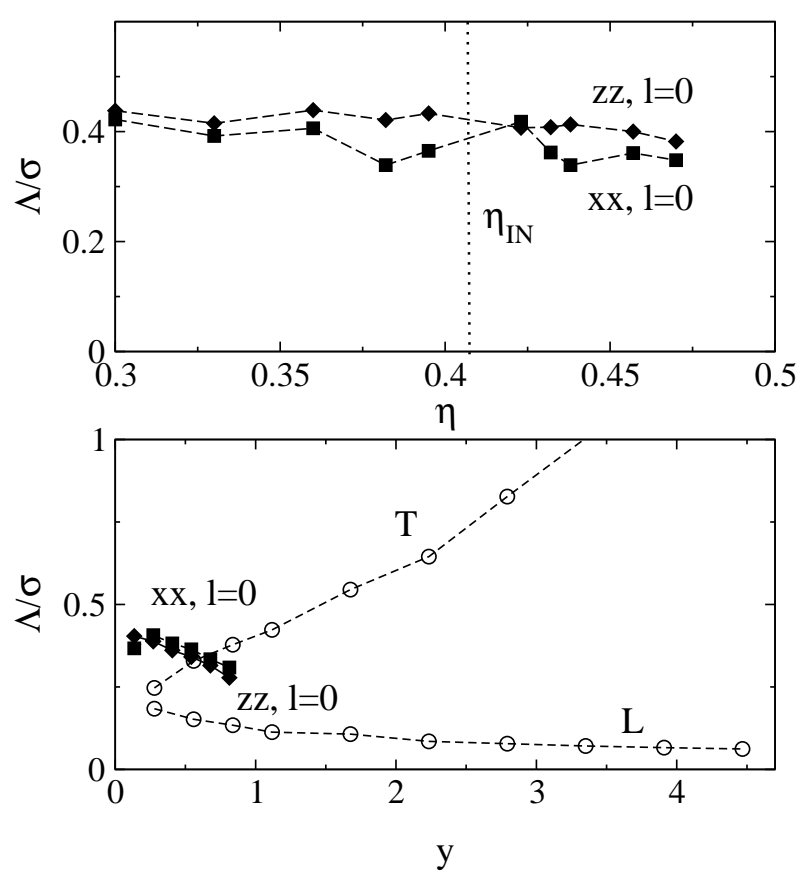

FIG. 3: The length of dipolar correlations in the fluid of hard spherocylinders vs the solvent packing density (upper panel, $\left(m^{*}\right)^{2}=1.0$ ) and vs dipolar density $y$ (lower panel, $\left.\eta=0.47\right)$. The data are obtained from NVT MC simulations. Closed diamonds refer to $z z, l=0$ projection, closed squares indicate $x x, l=0$ projection. Open points in the lower panel indicate the transverse and longitudinal correlation length obtained for a fluid of dipolar hard spheres with constant density $\rho \sigma^{3}=0.8$ and changing dipole moment. The dashed lines connect the simulation points. The dotted vertical tine in the upper panel indicates the density of the isotropic-nematic phase transition, $\eta_{\mathrm{IN}}=0.407$.

where $\tilde{h}^{l m n}(k)$ is the Hankel transform [Eq. A3 ] ]

In contrast to longitudinal and transverse projections of isotropic fluids, the projections $S_{\alpha \beta, l}(k)$ correspond to the same index $l$. The anisotropy of the nematic phase relative to the director is then taken out to the Legendre polynomial $P_{l}\left(\cos \theta_{k}\right)$ [Eq. (48)]. In addition, we found that $\Lambda_{l}$ calculated from different harmonics with the same $l$ are approximately equal to each other, at least for weak polar solvents. This is why $\Lambda_{l}$ in Eq. (56) does not include Cartesian projections $\alpha, \beta$. The results for $l=0$ are presented in Fig. 5] while data for $l>0$ do not converge well because of large statistical errors.

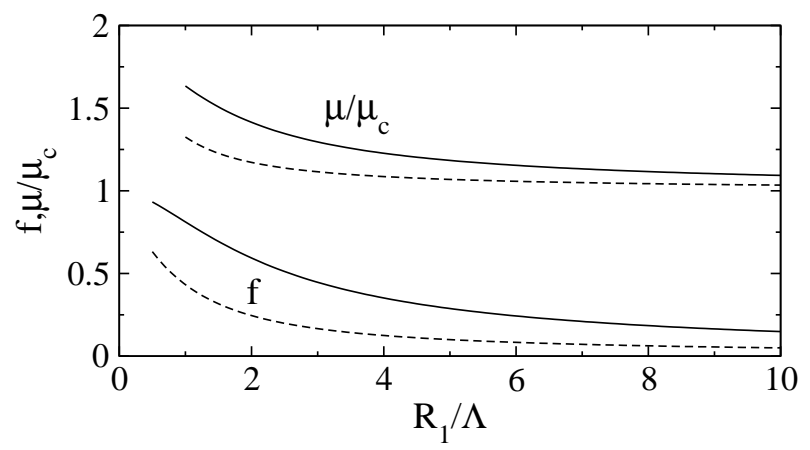

FIG. 4: Non-locality functions [Eq. [59] and reduced solvation free energy $\mu / \mu_{c}$ vs the solute size. The dashed and solid lines refer to the spherical ion and spherical dipole solutes, respectively.

With the structure factors given by Eq. (56), the solvation free energy is a linear combination of continuum and large wavevector limits:

$$
\mu=\mu_{c}+f\left(R_{1} / \Lambda\right)\left(\mu_{\infty}-\mu_{c}\right) .
$$

The function $f\left(R_{1} / \Lambda\right)$ represents the contribution of the non-local solvent response, influenced by the finite length of dipolar correlations, to the solvation thermodynamics. For the cases of spherical ionic (i) and dipolar (d) solutes, 
this function is given by the following expressions

$$
\begin{aligned}
& f^{(i)}(x)=0.5\left[1-e^{-2 x}\right] / x, \\
& f^{(d)}(x)=1.5\left[x^{2}-1+(x+1)^{2} e^{-2 x}\right] / x^{3} .
\end{aligned}
$$

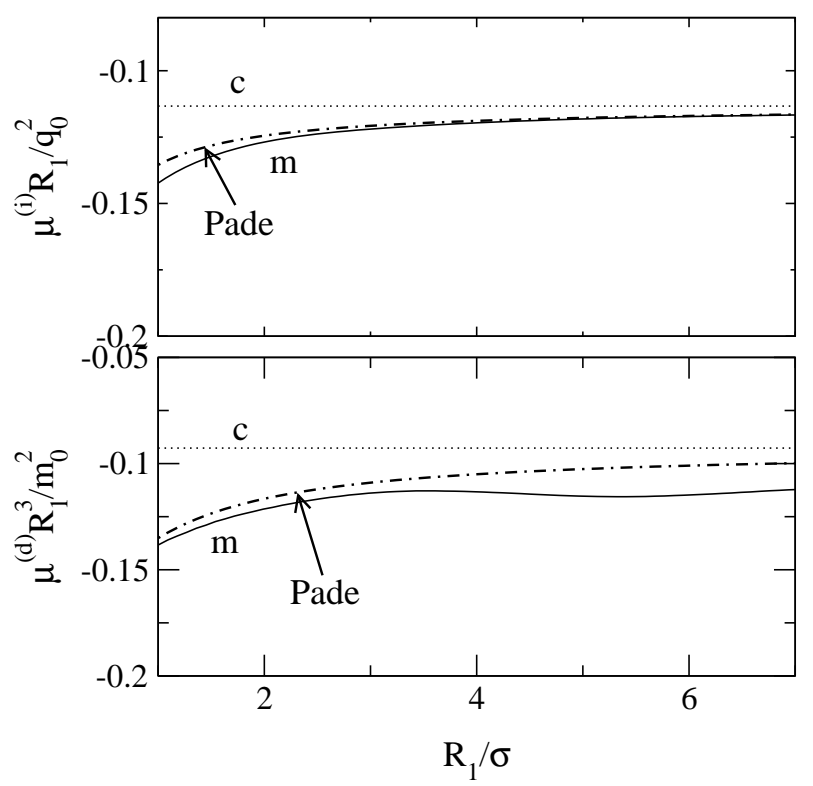

FIG. 5: Solvation free energy of ionic (upper panel, in $q_{0}^{2} / R_{1}$ units) and dipolar (lower panel, in $m_{0}^{2} / R_{1}^{3}$ units) solutes vs $R_{1}$. Shown are the microscopic calculation ["m", Eqs. [46- [48] ], the Padé form ["Pade", Eq. [58], and the continuum limit ["c", Eqs. (35) and (36)].

Non-locality functions $f^{(i)}\left(R_{1} / \Lambda\right)$ and $f^{(d)}\left(R_{1} / \Lambda\right)$ decay monotonically from one to zero with increasing the solute size $R_{1}$ (Fig. (4). The ionic non-locality function decays faster than the dipolar function indicating that ionic solvation is better described by continuum approximation than is dipolar solvation. Notice that formula (58) applies to solvation in strongly polar nematics, because it has been derived without assuming weak polarity of the solvent (the cross-terms omitted in Eq. (46) are included in $\mu_{\mathrm{c}}$ ). Figure 5 demonstrates a satisfactory agreement between the approximate solution given by Eq. [58) and the exact microscopic theory [Eqs. [46)-[48]]. The increase of the solvent dipole moment leads to an oscillatory character of the response function, but even in this case, the Padé approximation [Eq. (56)] gives reasonably accurate estimates for the solvation free energy in isotropic polar solvents $\underline{30}^{30}$

Equation (58), combining the limiting continuum and high wavevector values for ionic [Eqs. (35) and (54)] and dipolar [Eqs. (36) and (55)] solutes, is the central results of this study. The solvation free energy requires the following solvent parameters: dielectric constants $\epsilon_{\|}$and $\epsilon_{\perp}$, the order parameter $S_{2}$, the polarization correlation length $\Lambda$. In addition, the ionic charge or dipole moment, along with the radius $R_{1}=R_{0}+\sigma / 2$, should be supplied. The dielectric constants and the order parameter come from experiment. The correlation length $\Lambda$ is not experimentally available and, for the sake of interpreting the experiment, is, at the moment, a theory parameter requiring fitting to some experimental observable. Simulations of the model fluid of hard spherocylinders suggest magnitudes of $\Lambda$ of the order $\Lambda \simeq 0.3-0.4 \sigma$.

\section{THEORY PREDICTIONS}

The present theory allows us to make some specific predictions regarding equilibrium solvation and solvation dynamics. The electrostatic component of solvation free energy can be measured from the steady-state Stokes shift of optical lines, whereas solvation dynamics is probed by the Stokes shift correlation function. Our discussion below will therefore target these two properties. 


\section{A. Equilibrium solvation}

Our present development highlights several issues important for the understanding of equilibrium solvation in axially-symmetric solvents: (1) Solvation anisotropy, i.e. the dependence of the free energy of solvation on the orientation of the solute dipole relative to nematic director. (2) The effect of crossing the phase transition temperature on the solvation thermodynamics. (3) The effect of dipolar correlations on solvation and the applicability of continuum models of solvation.

From Eqs. (36), (55), and (58), the solvation free energy of a dipole, $\mu^{(d)}$, is a quadratic function of $\cos \theta_{0}$, where $\theta_{0}$ is the angle between the solute dipole and nematic director (Fig. 1). Figure 6) shows that the solvation anisotropy

$$
\Delta \mu^{(d)}=\mu_{\|}^{(d)}-\mu_{\perp}^{(d)}
$$

is negative in nematics with positive dielectric anisotropy $\left(\Delta \epsilon=\epsilon_{\|}-\epsilon_{\perp}>0\right)$ and positive otherwise $\left(\mu_{\|}^{(d)}=\mu^{(d)}\left(\theta_{0}=\right.\right.$ $0)$ and $\left.\mu_{\perp}^{(d)}=\mu^{(d)}\left(\theta_{0}=\pi / 2\right)\right)$. This can readily be verified by expanding Eq. (36) in powers of the small parameter $\Delta \epsilon$ :

$$
\mu_{\mathrm{c}}^{(d)} \approx-\frac{m_{0}^{2}}{2 R_{1}^{3}}\left[\frac{\epsilon_{s}-1}{2 \epsilon_{s}+1}+\frac{2 \Delta \epsilon}{5\left(2 \epsilon_{s}+1\right)^{2}} P_{2}\left(\cos \theta_{0}\right)\right]
$$

where

$$
\epsilon_{s}=\left(\epsilon_{\|}+2 \epsilon_{\perp}\right) / 3
$$

In order to relate anisotropy of $\mu_{\infty}^{(d)}$ to dielectric anisotropy, one needs a relation between the order parameter and the dielectric constants. This connection is given by the Maier-Meier theory: ${ }^{31}$

$$
\begin{aligned}
& \epsilon_{\|}-1=3 y \frac{3 \epsilon_{s}}{2 \epsilon_{s}+1}\left(1+2 S_{2} P_{2}(\cos \beta)\right), \\
& \epsilon_{\perp}-1=3 y \frac{3 \epsilon_{s}}{2 \epsilon_{s}+1}\left(1-S_{2} P_{2}(\cos \beta)\right) .
\end{aligned}
$$

From Eq. 63. $\Delta \epsilon \sim S_{2} P_{2}(\cos \beta)$. This means that both $\Delta \mu_{\infty}^{(d)}$ and $\Delta \mu^{(d)}$ change their sign from negative to positive when dielectric anisotropy changes its sign from positive to negative.

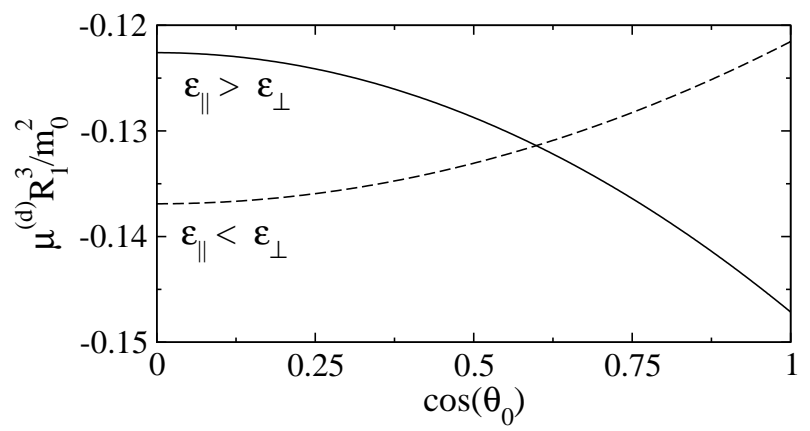

FIG. 6: The solvation free energy of a dipole solute (in $m_{0}^{2} / R_{1}^{3}$ units) vs the angle $\theta_{0}$ between the solute dipolar moment and the nematic director (Fig. (1); $R_{0}=1.7 \sigma$. Solid line refers to $\epsilon_{\|}>\epsilon_{\perp}$ (longitudinal solvent dipole, $\beta=0$ ) and the dashed line refers to $\epsilon_{\|}<\epsilon_{\perp}$ (transverse solvent dipole, $\beta=90$ ). Solvent parameters are: $\eta=0.47, S_{2}=0.8, y=0.15, \Lambda=0.3 \sigma$. At $\beta=0$, the dielectric anisotropy is positive, $\epsilon_{\perp}=1.092$ and $\epsilon_{\|}=2.63$; at $\beta=90^{\circ}$, the dielectric anisotropy is negative, $\epsilon_{\perp}=1.746$ and $\epsilon_{\|}=1.092$.

The magnitude of solvation anisotropy $\Delta \mu^{(d)}$ is strongly affected by the size of the solute. The continuum estimate of the solvation anisotropy parameter

$$
\kappa=\Delta \mu^{(d)} / \mu_{\mathrm{av}}^{(d)}, \quad \mu_{\mathrm{av}}^{(d)}=\frac{1}{3}\left(\mu_{\|}^{(d)}+2 \mu_{\perp}^{(d)}\right)
$$

gives very low anisotropies $(\kappa \simeq 0.02-0.03)$ for a large number of nematics $\frac{28}{2}$ Once the dipole correlation effects are involved through $\mu_{\infty}^{(d)}$, anisotropy becomes quite significant, $\kappa \simeq 0.3 S_{2}$. This solvation anisotropy results in a discontinuity of the solvation chemical potential at the point of the isotropic-nematic phase transition. 
The significant effect of dipolar correlations on the solvation thermodynamics is seen from comparison of lower and upper panels in Fig. 7 which shows steady-state Stokes shift of coumarin-153 dissolved in 4-n-pentyl-4'cyanobiphenyl $(5 \mathrm{CB})$ nematogen. This chromophore is widely used as a spectroscopic probe of solvation dynamics and thermodynamics 32.33 Stokes shift dynamics of coumarin-153 in the isotropic phase of a liquid crystalline solvent 11 and of coumarin-503.10 in both isotropic and nematic phases have been reported. The continuum limit in the lower panel reveals a much weaker anisotropy in the nematic phase than the full microscopic calculation in the upper panel. The continuum calculations are also much lower in the absolute magnitude, which is normally off-set by choosing the radius $R_{0}$ instead of the closest approach distance $R_{1}$ (Fig. 7 dash-dotted line). However, for the present calculation, re-scaling the cavity radius does not fully recover the solvation energy. This result suggests that polar nematics might produce stronger solvation than isotropic solvents with a comparable dielectric constant.

The electrostatic field of coumarin-153 is similar to that of a point dipole, 34 which makes it a convenient system to test our theory. Since the Stokes shift experiments measure only nuclear solvation, the Stokes shift $h c \Delta \bar{\nu}_{\mathrm{st}}(\bar{\nu}$ is the wavenumber, $\mathrm{cm}^{-1}$ ) was calculated according to the following expression

$$
h c \Delta \bar{\nu}_{\mathrm{st}}=-2 \mu_{n}^{(d)}\left(m_{0}=\Delta m_{0}\right) .
$$

Here, the difference in the dipole moments in the excited and ground states of the chromophore $\Delta m_{0}$ is substituted for the solute dipole moment. The nuclear component of solvation is calculated in the additive approximation ${ }^{35}$ in which the overall solvation free energy in a polar/polarizable liquid is assumed to be the sum of the nuclear and electronic solvation components. The overall solvation free energy $\mu\left(\epsilon_{\|}, \epsilon_{\perp}, y_{\text {eff }}\right)$ is calculated from the anisotropic dielectric constant in the component $\mu_{c}\left(\epsilon_{\|}, \epsilon_{\perp}\right)$ and the effective dipolar density $y_{\text {eff }}$ in $\mu_{\infty}\left(y_{\text {eff }}\right)$. The effective dipolar density is defined as ${ }^{36}$

$$
y_{\mathrm{eff}}=\left(4 \pi / 9 k_{\mathrm{B}} T\right) \rho\left(m^{\prime}\right)^{2}+(4 \pi / 3) \rho \alpha,
$$

where $\alpha$ is the dipolar polarizability and $m^{\prime}$ is the average dipole moment of the solvent in the liquid. The nuclear free energy of solvation is then given by

$$
\mu_{n}=\mu\left(\epsilon_{\|}, \epsilon_{\perp}, y_{\mathrm{eff}}\right)-\mu\left(n_{\|}^{2}, n_{\perp}^{2}, y_{e}\right)
$$

where $n_{\perp, \|}$ is the anisotropic refractive index and the density of induced dipoles is

$$
y_{e}=(4 \pi / 3) \alpha \rho .
$$

For coumarin-153, the radius $R_{0}=4.89 \AA$ and the dipole moment change $\Delta m_{0}=7.53 \mathrm{D}$ have been adopted ${ }^{37}$

$5 \mathrm{CB}$ was chosen as a typical nematogen with its physical properties well documented in the literature: the isotropicnematic transition temperature, $T_{\mathrm{IN}}=308.2 \mathrm{~K}^{38}$, dipole moment, $m=4.75 \mathrm{D}, 39$ and the azimuthal angle between the dipole moment and the long axis, $\beta=21.6^{0} \underline{\underline{40}}$ The temperature dependences of the static dielectric constants $\underline{39.40}$ refraction indexes ${ }^{39.41}$ the order parameter ${ }^{41}$ and density ${ }^{39}$ have also been reported. For the solvent diameter the value for benzene, $\sigma=5.27 \AA ⿻ 37$ was adopted, and the polarization correlation length was calculated from Fig. B as $\Lambda=0.35 \sigma$ corresponding to $\left(m^{*}\right)^{2}=3.3-3.6$. The dipole moment $m^{\prime}$ was calculated using the Onsager approximation

$$
m^{\prime}=\frac{\left(n^{2}+2\right)\left(2 \epsilon_{s}+1\right)}{3\left(2 \epsilon_{s}+n^{2}\right)} m,
$$

where $\epsilon_{s}$ is given by Eq. (62) and $n^{2}=\left(n_{\|}^{2}+2 n_{\perp}^{2}\right) / 3$.

Note that this approximation is made in the Maier-Meier theory [Eq. [63] ] which, nevertheless, describes dielectric properties of polar nematics reasonably well ${ }^{42.43}$ All results in Fig. [7 have been obtained at experimentally documented parameters of $5 \mathrm{CB}$, the gap between the Stokes shift curves around the isotropic-nematic transition temperature reflects the absence of experimental data in this temperature range.

\section{B. Stokes shift dynamics}

The dynamics of solvation following the photoinduced change in the solute charge distribution is recorded by measuring the Stokes shift correlation function ${ }^{44}$

$$
S(t)=\frac{E(t)-E(\infty)}{E(0)-E(\infty)},
$$




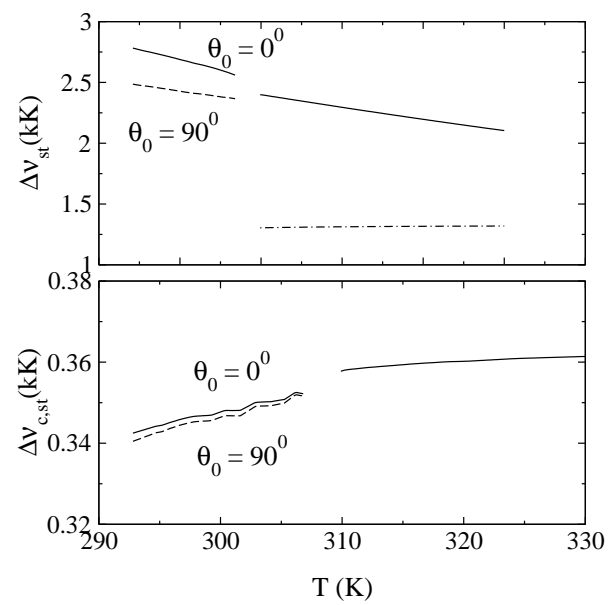

FIG. 7: Stokes shift of coumarin-153 in nematic and isotropic phases of 5CB. The upper panel shows the microscopic calculation according to Eq. (58), the lower panel shows the continuum limit. The solid lines refer to the parallel alignment of the solute dipole with the nematic director, the dashed lines refer to the perpendicular alignment [Fig. 1. The solute radius and the dipole moment are $R_{0}=4.89 \AA$ and $\Delta m_{0}=7.5 \mathrm{D}$, respectively. The temperature dependent dielectric constants, ${ }^{39.41}$ order parameter, ${ }^{41}$ and density ${ }^{39}$ of $5 \mathrm{CB}$ are taken from experiment; $\Lambda=0.35 \sigma$. The dash-dotted line in the upper panel refers to the continuum isotropic result calculated with the cavity radius equal to $R_{0}$.

where $E(t)$ is the time-dependent energy of the solute. The calculation of this function is normally accomplished within the linear response theory. Several formulations of the theory are available in the literature, ${ }^{45,46,47}$ and we adopt here the formulation due to Wolynes $\frac{45}{}$ which represents the Laplace transform $E(s)$ of the time-dependent function $E(t)$ as the equilibrium solvation energy characterized by the dielectric constant $\epsilon(s)$

$$
E(s)=E\left(\epsilon_{\|}(s), \epsilon_{\perp}(s)\right)=\frac{2}{s}\left(\mu\left(\epsilon_{\|}(s), \epsilon_{\perp}(s), y_{\mathrm{eff}}\right)-\mu\left(n_{\|}^{2}, n_{\perp}^{2}, y_{e}\right)\right) .
$$

The dependence of $\Lambda$ on the dielectric constant of a nematic solvent is generally unknown. We will therefore assume this parameter independent of the Laplace variable $s$. Within this approximation, the Stokes shift function is fully determined by the continuum expression for the solvation energy [Eq. [36)]. The results for $S(t)$ presented here are therefore based on the continuum solution for the dipolar solvation energy in an anisotropic dielectric with axial symmetry.

Experimental dielectric data on nematics are well characterized by the two-exponential form

$$
\epsilon_{a}(s)=\epsilon_{\infty a}+\left(\epsilon_{0 a}-\epsilon_{\infty a}\right) \sum_{k=1,2} \frac{g_{k}}{1+s \tau_{k a}},
$$

where $g_{1}+g_{2}=1$ and $a$ stands for $\|, \perp$ or $i s o$ (isotropic). This form reflects two different relaxations: high-frequency rotation around a long molecular axis (relaxation time $\tau_{2}$ ) and low-frequency rotation around a short molecular axis (relaxation time $\left.\tau_{1}\right)^{38}$ In isotropic phase, $\tau_{2 i s o}$ is about ten times smaller that $\tau_{1 i s o}$, and the contribution from highfrequency rotation to dielectric loss is usually small and is rarely resolved in the dielectric experiments ${ }^{28.38}$ With only one relaxation time $\tau_{1 i s o}$, Eq. (72) reduces to the Debye dispersion resulting in a single-exponential Stokes shift correlation function with the relaxation time $\tau_{S}=\left[\left(2 \epsilon_{\infty}+1\right) /\left(2 \epsilon_{0}+1\right)\right] \tau_{1 i s o}$ (Ref. 47).

The liquid crystalline order of the nematic phase hinders rotations around a short molecular axis when it is perpendicular to the nematic director. The corresponding relaxation time $\tau_{1 \|}$ is about ten times larger than $\tau_{1 i s o}$. In contrary, $\tau_{1 \perp}$ is much smaller than $\tau_{1 i s o}$, and can be comparable to $\tau_{2 a}(a=\|, i s o) \underline{28}$

With the dielectric constant from Eq. (72) substituted into Eq. (36) the function $S(t)$ needs to be calculated numerically. Following the procedure described in Ref. 48, the function $F(s)=-s E(s)$ was fitted to a sum of Cole-Davidson type functions

$$
F(s)=(E(0)-E(\infty)) \sum_{i} \frac{a_{i}}{\left(1+s \tau_{S i}\right)^{\gamma_{i}}}
$$

with the exponents $\gamma_{i}$ and Stokes shift relaxation times $\tau_{S i}$; the linear expansion coefficients are normalized by the 
condition $\sum_{i} a_{i}=1$. Equation (73) allows analytical inverse Laplace transform:

$$
S(t)=\sum_{i} a_{i} \frac{\Gamma\left(\gamma_{i}, t / \tau_{S i}\right)}{\Gamma\left(\gamma_{i}, 0\right)}
$$

where $\Gamma(\gamma, t)$ is the incomplete gamma function. $\underline{49}$

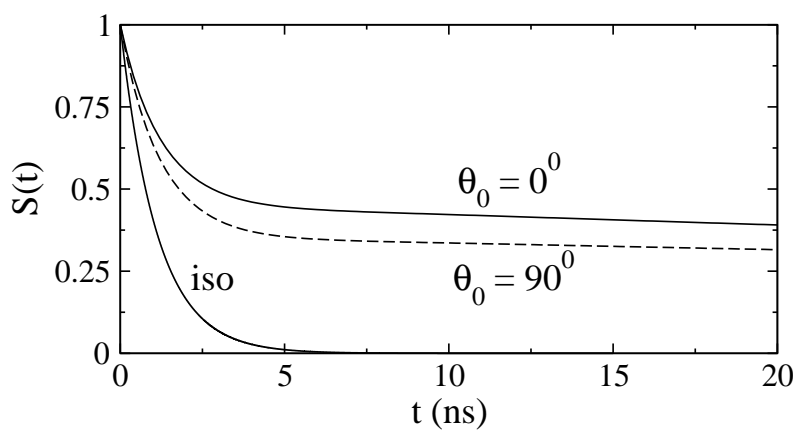

FIG. 8: Stokes shift correlation function in the isotropic phase ("iso") and in the nematic phase at two orientations of the solute relative to the nematic director.

Function $S(t)$ calculated for a spherical dipolar dye in 4,4-n-heptyl-cyanopiphenyl (7CB) is presented in Fig. 8 Experimental data for dielectric constants, refractive indexes and low-frequency relaxation times is taken from Ref. 50. No high-frequency relaxation times $\tau_{2 a}$ have been reported for this nematogene. $S(t)$ in the isotropic phase (line marked "iso" in Fig. [8) is single-exponential, as expected.

Two predictions follow from our calculations in the nematic phase. First, $S(t)$ is bi-exponential. The slow component with $\tau_{S 1} \approx 100-500 \mathrm{~ns}$ and $\gamma_{1} \approx 0.82$ is related to $\epsilon_{\|}$, and the fast component with parameters $\tau_{S 2} \approx 1-2 \mathrm{~ns}$ and $\gamma_{2} \approx 1.2$ is related to the relaxation of $\epsilon_{\perp}$. Second, $S(t)$ is effected by the angle $\theta_{0}$ between the solute dipole and the nematic director. For nematics with positive dielectric anisotropy (e.g., $7 \mathrm{CB}) S(t)$ decays faster for $\theta_{0}=90^{0}$ than for $\theta_{0}=0^{0}$. Note that the slow component has not been detected in experimental studies of transient Stokes shift in nematics ${ }_{9.10 .11}$ probably because of the limited experimental time resolution (a few ns). All Stokes shift relaxation times monotonically increase with lowering temperature in both the nematic and isotropic phase (Fig. 9).

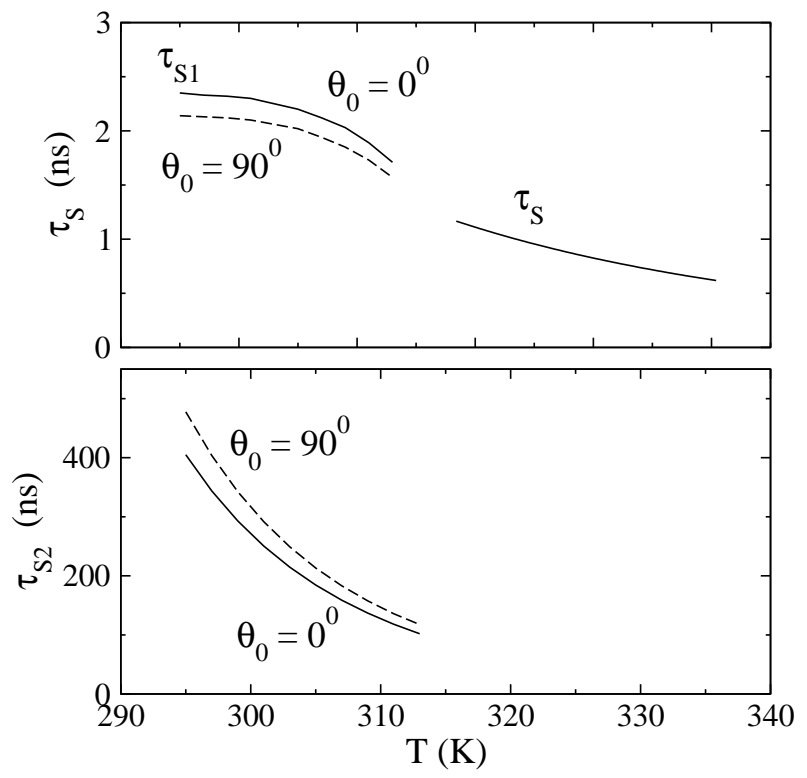

FIG. 9: Temperature dependence of the Stokes shift relaxation times. The upper panel shows the faster relaxation time in the isotropic $\left(\tau_{S}\right)$ and nematic $\left(\tau_{S 2}\right)$ phases. The lower panel shows the slower relaxation time $\left(\tau_{S 1}\right)$ present only below $T_{\mathrm{IN}}$. The solid lines refer to the orientation of the solute dipole parallel to the nematic director, while the dashed lines refer to the perpendicular orientation. 


\section{CONCLUSIONS}

This article presents a microscopic theory of solvation in solvents with axial symmetry. Although applications of the theory considered here are limited to nematic liquid crystals, i.e. liquids with inversion symmetry of the polar axis, the formalism is also applicable to ferroelectric solvents with a preferential polar direction. This conclusion follows from the fact that the linear response approximation leads to a quadratic dependence on the solute dipole moment invariant to the dipole flip. For ferroelectrics, however, the solvation energy gains an additional contribution, linear in the solute dipole, from the macroscopic polarization of the solvent.

The full microscopic formulation requires $k$-dependent dipolar susceptibility of the nematic solvent which needs to be obtained from computer experiment. From the analysis of the results of MC simulations and the microscopic formalism, we have derived a formula for the solvation chemical potential which is based on experimentally measurable input parameters and a theory parameter, the length of dipolar correlations in the solvent. This correlation length was obtained from MC simulations in the range of parameters attainable for the model fluid of dipolar spherocylinders. The understanding of the properties of the correlation length in a broader range of parameters, in particular for higher dielectric constants, will require simulations of nematogenes with more realistic intermolecular potentials.

The theory provides a quantitative framework for interpreting the spectroscopic steady-state and time-resolved experiments and makes several experimnetally testable predictions. We show that the equilibrium free energy in the nematic phase is a quadratic function of cosine of the angle between the solute dipole and the solvent nematic director. The sign of solvation anisotropy is determined by the sign of dielectric anisotropy of the solvent: solvation anisotropy is negative in solvents with positive dielectric anisotropy and vice versa. The solvation free energy is discontinuous at the point of isotropic-nematic transition. The amplitude of this discontinuity is strongly affected by the size of the solute becoming less pronounced for larger solutes. The discontinuity itself and the magnitude of the splitting of the solvation chemical potential in the nematic phase are mostly affected by microscopic dipolar correlations in the nematic solvent.

The Stokes shift correlation function in the isotropic phase is one-exponential when dielectric relaxation is given by the Debye form. The corresponding relaxation time is a smooth function of temperature through isotropic-nematic transition. In the nematic phase, the Stokes shift relaxation becomes bi-exponential with a much slower relaxation component related to rotations of polar molecules around their short axes in the nematic potential strongly hindering such motions.

\section{APPENDIX A: DERIVATION OF EQ. (27)}

We start with the formal expression for the solvent dipolar susceptibility ${ }^{23}$ :

$$
\begin{aligned}
\tilde{\chi}_{s}(\mathbf{k})= & \left(m^{2} / k_{\mathrm{B}} T\right) \int d \omega_{1} d \omega_{2} \\
& \hat{\mathbf{m}}\left(\omega_{1}\right) \hat{\mathbf{m}}\left(\omega_{2}\right)\left[\delta\left(\omega_{1}-\omega_{2}\right) \rho\left(\omega_{1}\right)+\rho\left(\omega_{1}\right) \rho\left(\omega_{2}\right) \tilde{h}\left(\mathbf{k}, \omega_{1}, \omega_{2}\right)\right],
\end{aligned}
$$

where $m$ and $\omega$ are the magnitude and orientation of the solvent dipole. Further, the pair correlation function $\tilde{h}\left(\mathbf{k}, \omega_{1}, \omega_{2}\right)$ is expanded in spherical harmonics ${ }^{22.23}$

$$
\tilde{h}\left(\mathbf{k}, \omega_{1}, \omega_{2}\right)=\sum_{l_{1} l_{2} l} \sum_{n_{1} n_{2}} \tilde{h}_{n_{1} n_{2}}^{l_{1} l_{2} l}(k) Y_{l_{1}, n_{1}}\left(\omega_{1}\right) Y_{l_{2}, n_{2}}\left(\omega_{2}\right) Y_{l, n_{1}+n_{2}}^{*}\left(\omega_{k}\right)
$$

where $\tilde{h}_{n_{1} n_{2}}^{l_{1} l_{2} l}(k)$ is the Hankel transform:

$$
\tilde{h}_{n_{1} n_{2}}^{l_{1} l_{2} l}(k)=4 \pi i^{l} \int_{0}^{\infty} d r r^{2} j_{l}(k r) h_{n_{1} n_{2}}^{l_{1} l_{2} l}(r) .
$$

Then the tensor $\tilde{\chi}_{s}$ can be written as:

$$
\tilde{\chi}_{s, n_{1} n_{2}}(\mathbf{k})=\sum_{l} \tilde{\chi}_{s, n_{1} n_{2} l}(k) Y_{l,-n_{1}-n_{2}}^{*}\left(\omega_{k}\right)
$$

where coefficients $\tilde{\chi}_{s, n_{1} n_{2} l}(k)$ are proportional to $\tilde{h}_{n_{1} n_{2}}^{l_{1} l_{2} l}(k)$ and depend only on the magnitude of wave-vector $k$. 
We next prove the relation

$$
\tilde{\chi}_{s}(k=0)-\tilde{\chi}^{\prime}(k=0)=\int \frac{d \omega_{k}}{4 \pi} \tilde{\chi}_{s}(k=0)
$$

where $\tilde{\chi}^{\prime}$ is the part of $\tilde{\chi}_{s}$ which arises from solvent occupied the volume outside of the solute. Since the function $\tilde{\chi}^{\prime}$ is defined by integrating over the volume twice larger than the solute volume it depends only on the asymptote of solvent correlation function on long distances. We will seek the asymptotes of $h_{n_{1} n_{2}}^{l_{1} l_{2} l}(r)$ in form $1 / r^{n}$. Since $h(12)$ cannot decay slower than the interaction potential, $n \geq 3$. According to (A4) and (A3) the contribution from the asymptotes is proportional to

$$
I=k^{n-3} \int_{k d}^{\infty} d x \frac{j_{l}(x)}{x^{n-2}}
$$

where $d$ is an arbitrary length larger than size of solvent particle.

We first consider the case $n=3$ :

$$
I=\int_{k d}^{\infty} d x \frac{j_{l}(x)}{x}
$$

In the limit $k \rightarrow 0$, this integral converges only if $l>0$. Since $\tilde{\chi}_{s}(\mathbf{k})$ is analytical function at $k=0$, only harmonics with $l>0$ can have asymptote $1 / r^{3}$. In case of $n>3$ we split $I$ into two parts:

$$
I=k^{n-3} \int_{\delta}^{\infty} d x \frac{j_{l}(x)}{x^{n-2}}+k^{n-3} \int_{k d}^{\delta} d x \frac{j_{l}(x)}{x^{n-2}},
$$

where parameter $\delta$ is small enough that $j_{l}(x)$ can be replaced by the first term of its Taylor expansion $x^{l} /(2 l+1) !$ ! Then the first part of $I$ vanishes in limit $k \rightarrow 0$. The second part is proportional to

$$
k^{n-3} \int_{k d}^{\delta} \frac{x^{l-n+2}}{(2 l+1) ! !} \sim k^{l}
$$

and in the limit $k \rightarrow 0$ gives non-zero contribution only into term with $l=0$. Therefore, in long-wave limit term $l=0$ in Eq. (A4) arises from harmonics with asymptotes $1 / r^{n}$ where $n>3$, and all therms $l>0$ steam from harmonics with asymptotes $1 / r^{3}$. Assuming that only the $1 / r^{3}$ asymptotes contribute to $\tilde{\chi}^{\prime}(k=0)$, we arrive in Eq. A5 from Eq. (A4) and orthogonality of spherical harmonics.

\section{ACKNOWLEDGMENTS}

This research was supported by the National Science Foundation (CHE-0304694). This is publication \#646 from the ASU Photosynthesis Center.

1 M. Born, Z. Phys. 1, 45 (1920).

2 L. Onsager, J. Am. Chem. Soc. 58, 1486 (1936).

3 J. G. Kirkwood, J. Chem. Phys. 2, 351 (1934).

4 C. J. Cramer and D. G. Truhlar, Chem. Rev. 99, 2161 (1999).

5 J. Tomasi, Theor. Chem. Acc. 112, 184 (2004).

${ }^{6}$ F. O. Raineri and H. L. Friedman, Adv. Chem. Phys. 107, 81 (1999).

7 J. Richardi, P. H. Fries, and H. Krinke, J. Chem. Phys. 108, 4079 (1998).

8 T. Urisu, K. Kajiyama, and Y. Mizushima, Appl. Optics 17, 2366 (1978).

${ }^{9}$ G. Saielli, A. Polimeno, P. L. Nordio, P. Bartolini, M. Ricci, and R. Righini, J. Chem. Soc. Faraday Trans. 94, 121 (1998).

10 P. Bartolini, M. Ricci, R. Righini, G. Saielli, A. Polimeno, and P. L. Nordio, Mol. Cryst. Liq. Cryst. 336, 33 (1999). 
11 J. Rau, C. Ferrante, E. Kneuper, F. W. Deeg, and C. Bräuchle, J. Phys. Chem. A 105, 5734 (2001).

12 M. C. B. Mennucci and J. Tomasi, J. Chem. Phys. 102, 6837 (1995).

13 E. C. B. Mennucci and J. Tomasi, J. Phys. Chem. B 101, 10506 (1997).

14 M. Inoue and K. Urano, J. Chem. Phys. p. 3672 (1975).

15 K. Urano and M. Inoue, J. Chem. Phys. 66, 791 (1977).

16 D. V. Matyushov, J. Chem. Phys. 120, 1375 (2004).

17 P. de Gennes, The physics of liquid crystals (Clarendon press, Oxford, 1974).

18 D. Chandler, Phys. Rev. E 48, 2898 (1993).

19 C. J. F. Böttcher, Theory of Electric Polarization, vol. 1 (Elsevier, 1973).

20 X. Song, D. Chandler, and R. A. Marcus, J. Phys. Chem. 100, 11954 (1996).

21 J. P. Hansen and I. R. McDonald, Theory of Simple Liquids (Academic Press, 2003).

22 C. G. Gray and K. E. Gubbins, Theory of Molecular Liquids (Clarendon Press, Oxford, 1984).

23 S. H. L. Klapp and G. N. Patey, J. Chem. Phys. 112, 3832 (2000).

24 J. W. J.M. Caillol and G. Patey, Phys. Rev. A 38, 4772 (1988).

${ }^{25}$ L. Landau and E. Lifshitz, Electrodynamics of continuous media (Pergamon press, Oxford, 1984).

${ }^{26}$ M. Lilichenko and D. V. Matyushov, J. Chem. Phys. 119, 1559 (2003).

27 S. C. McGrother, A. Gil-Villegas, and G. Jackson, Mol. Phys. 95, 657 (1998).

28 D. Dunmur, A. Fukuda, and G. Luckhurst, eds., Physical properties of Liquid Crystals: Nematics (Exeter, 2001).

${ }^{29}$ H. E. Stanley, Introduction to phase transitions and critical phenomena (Oxford University Press, New York, 1987).

30 D. V. Matyushov, Mol. Phys. 79, 795 (1993).

31 W. Maier and G. Meier, Z. Naturforsch. 16a, 262 (1961).

32 M. Maroncelli, J. Mol. Liq. 57, 1 (1993).

33 L. Reynolds, J. A. Gardecki, S. J. V. Frankland, and M. Maroncelli, J. Phys. Chem. 100, 10337 (1996).

34 P. V. Kumar and M. Maroncelli, J. Chem. Phys. 103, 3038 (1995).

35 S. Gupta and D. V. Matyushov, J. Phys. Chem. A 108, 2087 (2004).

36 G. Stell, G. N. Patey, and J. S. Høye, Adv. Chem. Phys. 18, 183 (1981).

37 D. V. Matyushov and M. D. Newton, J. Phys. Chem. A 105, 8516 (2001).

38 S. Urban, B. Gestblom, and A. Würflinger, Mol. Cryst. Liq. Cryst. 331, 113 (1999).

39 S. Urban, B. O. Gestblom, and R. Dabrowski, Phys. Chem. Chem. Phys. 1, 4843 (1999).

40 J. K. S. Urban and R. Dabrowski, Z. Naturforschg. 55a, 449 (2000).

41 R. Horn, Le Journal de Physique 39, 105 (1978).

${ }^{42}$ H. Kresse, in Advances in Liquid Crystals, edited by G. H. Brown (Academic Press, 1983), vol. 6, p. 109.

43 T. K. Bose, B. Campbell, S. Yagihara, and J. Thoen, Phys. Rev. A 36, 5767 (1987).

44 M. Maroncelli and G. R. Fleming, J. Chem. Phys. 86, 6221 (1987).

45 P. G. Wolynes, J. Chem. Phys. 86, 5133 (1987).

46 L. E. Fried and S. Mukamel, J. Chem. Phys. 93, 932 (1990).

47 B. Bagchi and A. Chandra, Adv. Chem. Phys. 80, 1 (1991).

48 D. V. Matyushov, J. Chem. Phys. 122, 044502 (2005).

49 I. S. Gradshteyn and Ryzhik, Table of Integrals, Series, and Products (Academic Press, San Diego, 1994).

${ }^{50}$ M. Davies, R. Moutron, P. A, H, M. S. Beevers, and J. Williams, J. Chem. Soc. Faraday Trans. 2 72, 1447 (1976). 